Mots. Les langages du politique

\title{
Lionel Meney, Main basse sur la langue. Idéologie et interventionnisme linguistique au Québec
}

2010, Montréal, Liber, 510 pages

Albane Geslin

\section{OpenEdition}

\section{Journals}

Édition électronique

URL : https://journals.openedition.org/mots/20787

DOI : $10.4000 /$ mots. 20787

ISSN : 1960-6001

Éditeur

ENS Éditions

Édition imprimée

Date de publication : 15 septembre 2012

Pagination : 143-146

ISBN : 9782847883732

ISSN : 0243-6450

\section{Référence électronique}

Albane Geslin, «Lionel Meney, Main basse sur la lanque. Idéologie et interventionnisme linguistique au

Québec », Mots. Les langages du politique [En ligne], 99 | 2012, mis en ligne le 15 septembre 2014 consulté le 22 avril 2022. URL : http://journals.openedition.org/mots/20787 ; DOI : https://doi.org/ $10.4000 /$ mots. 20787 


\title{
Comptes rendus de lecture
}

\author{
Main basse sur la langue. Idéologie et \\ interventionnisme linguistique au Québec \\ Lionel Meney \\ 2010, Montréal, Liber, 510 pages
}

Le titre et le sous-titre donnent, dès l'abord, le ton de l'ouvrage. Lionel Meney signe en effet un vigoureux réquisitoire, confinant parfois à la diatribe, à l'encontre de ceux - qualifiés d'« endogénistes » - qui promeuvent un interventionnisme étatique québécois visant à (im) poser la norme linguistique d'un « français québécois standard », autonome à l'égard du français standard international.

Dès les premières pages de ce livre d'une grande densité, mais non dénué d'humour, le débat est posé : s'il y a consensus pour reconnaître que le français est une langue sur la défensive au Québec, les avis divergent quant au modèle linguistique à promouvoir. Doit-on adopter une norme "endogène», s'appuyant sur l'espace francophone québécois et, dans l'affirmative, la norme doit-elle s'aligner sur la langue du peuple ou sur celle de l'élite, ce qui nécessite de poser la question de la légitimité du choix? Ou doit-on s'aligner sur un modèle linguistique transnational, celui du français standard international tel qu'il est parlé et écrit par les locuteurs francophones instruits quel que soit leur rattachement géographique? La première option suppose un interventionnisme étatique important, la seconde non.

Radicalement opposé aux thèses endogénistes, Lionel Meney va, dans un premier temps, présenter, à grand renfort de statistiques démolinguistiques parfois roboratives, le cadre géopolitique et linguistique dans lequel s'insère la nation québécoise, afin de mesurer la place géopolitique du Québec et le poids des francophones canadiens dans le marché linguistique mondial et le marché linguistique francophone. Cette analyse lui permet d'affirmer que le français au Québec est concurrencé par l'anglais (l'américain plus précisément, de plus en plus parlé par les anglophones du Québec et les allophones). Ces études lui permettent en outre d'affirmer que, contrairement à ce que soutiennent les partisans d'une norme linguistique québécoise autonome, « la tendance générale du marché linguistique québécois va dans le sens d'une convergence toujours plus grande avec le marché francophone international» (p.131).

L'auteur ne nie pas que le parlé québécois présente des particularités au regard du français standard. Il en fait une présentation claire : que ce soit les anglicismes propres au français québécois (par emprunt de termes ou de sens, 
par calques...; par ex. billet de saison), les conservatismes (termes issus des dialectes français mais pour ainsi dire disparus du français standard : crochir pour tordre), les rares emprunts aux langues amérindiennes ou les néologismes (tel que balayeuse pour aspirateur). Cependant, contrairement aux endogénistes, il n'analyse pas ces «québécismes » comme justifiant d'élaborer une norme propre aux Québécois, mais comme un phénomène de «diglossie », à savoir la coexistence, depuis environ deux siècles, dans la société québécoise, de deux systèmes linguistiques. L'un vernaculaire, le "franbécois », non standardisé, ni dans sa grammaire ni dans son orthographe, mais jouant un rôle très important dans la communication quotidienne de la majorité des Québécois (mais très difficile à comprendre pour des locuteurs francophones non québécois); c'est une langue populaire, orale comme écrite. L'autre système est le français standard, qui s'apprend à l'école, largement utilisé dans les médias, en politique, en littérature... Ainsi, mis à part ces québécismes, la langue des Québécois ne se différencie pour ainsi dire pas du français standard, tant du point de vue phonologique, morphologique, syntaxique que lexical. Les endogénistes considèrent, quant à eux, que les particularités québécoises révèlent deux niveaux de langue (familier-populaire / neutre-soutenu) dans un système linguistique unique, le « français québécois standard », dont il faut officialiser et diffuser la norme - le français « tel qu'il est écrit par l'élite québécoise, qui possède un français de qualité » (Lionel Meney citant ici deux des auteurs du rapport Larose, p. 298) -, norme qui doit être observée non seulement par l'administration, mais également par la société civile.

L'ouvrage va, par la suite (troisième et quatrième parties), changer de ton pour se faire plus agressif et incisif, et présenter les travaux des linguistes endogénistes. Différents rapports, études et dictionnaires vont ainsi être impitoyablement passés au crible de l'analyse du linguiste et lexicographe qu'est Lionel Meney. Ainsi en est-il de l'ouvrage de Christiane Loubier, Les emprunts. Traitement en situation d'aménagement linguistique ${ }^{1}$, du fameux rapport Larose, Le français, une langue pour tout le monde ${ }^{2}$, du Dictionnaire québécois d'aujourd'huis ou du Grand dictionnaire terminologique4, pour ne citer qu'eux. L'auteur, moult exemples à l'appui, dresse le tableau critique de ces ouvrages: peu convaincants, contradictoires, trahissant des conflits d'intérêts, erronés, manquant de rigueur, traitant de façon critiquable les anglicismes, partiaux, dénotant une méconnaissance du « français de France » et ne maîtrisant pas le français québécois standard dont les auteurs prônent l'officialisation, faisant

1. Québec, Office québécois de la langue française, 2003.

2. Rapport de la Commission des États généraux sur la situation et l'avenir de la langue française au Québec, 20 août 2001, en ligne (format PDF) : [http://www.spl.gouv.qc.ca/fileadmin/medias/ pdf/COM1-021_Rapport_final.pdf], consulté le 3 avril 2012.

3. Jean-Claude Boulanger éd., Paris, Le Robert, 1992.

4. Québec, Office québécois de la langue française, en ligne [http://www.oqlf.gouv.qc.ca/ressources/gdt.html], consulté le 3 avril 2012. 
usage de langue de bois, pour ne citer que les principaux reproches formulés. Les longs développements précédents (première et deuxième parties) analysant les statistiques sociolinguistiques et les sondages prennent alors tout leur sens, car ils permettent à l'auteur de se démarquer des travaux élaborés par les théoriciens endogénistes qui, le plus souvent, ne s'appuient sur aucun chiffre tout en affirmant que la norme qu'ils entendent décrire fait l'objet d'un consensus au sein de la société québécoise, consensus que Lionel Meney qualifie de «mythe» (p.372). L'on pourra cependant regretter quelques critiques «gratuites» de la part de l'auteur, notamment le fait qu'il demeure des coquilles dans le Dictionnaire québécois d'aujourd'hui (on soulignera que des fautes de frappe sont également présentes dans l'ouvrage de L. Meney, ce qui n'altère nullement la qualité du propos; on notera également que, dans le deuxième chapitre, l'auteur confond très fréquemment une variation en termes de pourcentage et une variation en termes de point, ce qui est quelque peu gênant).

Au-delà de la question linguistique et de ce qui peut sembler être, de l'extérieur, des querelles de chapelles, Lionel Meney propose toutefois un ouvrage véritablement politique. Il aborde ainsi la question des colonialismes (français, puis anglais) subis par les Québécois, mais également celle du colonialisme des Québécois à l'égard des peuples autochtones (faisant l'objet d'une "amnésie historique », p. 203), la question du soi-disant colonialisme linguistique et culturel de la France aujourd'hui et le problème de la francophobie animant certains endogénistes. Le discours anti-français, voire anti-parisien, est contre-productif car il se trompe de cible : la menace vient de l'anglais et non du français de France. En outre, contrairement à l'anglais ou à l'espagnol, pour lesquels les locuteurs sont beaucoup plus nombreux dans les anciennes colonies que dans les anciennes métropoles, la norme du français international réside, c'est un fait, en France, qui se trouve être le plus grand pays francophone.

L'auteur adresse aux endogénistes (et non seulement à leurs travaux) des critiques très dures : ils sont dans un déni de réalité (à l'égard du phénomène de diglossie notamment), sont promoteurs d'un protectionnisme linguistique qui risque d'isoler les Québécois des autres locuteurs francophones, sont ethnocentristes et nationalistes, ont une conception technocratique de la langue soutenant une ingérence de l'État et des spécialistes (que l'auteur qualifie de « linguocrates», p. 491) dans la formation et l'évolution linguistique. Ils font en outre de la langue un enjeu identitaire, le marqueur de la nation québécoise, alors que c'est avant tout un outil de communication. Ce que conteste Lionel Meney, c'est la volonté d'uniformiser la langue du Québec au détriment de l'intercompréhension des francophones, mais également au détriment de la société civile.

Mais l'auteur ne limite pas ses propos à la seule critique. Il propose, à la toute fin de sa conclusion, l'élaboration d'une nouvelle politique linguistique (et non pas une norme linguistique) : «ll faut détechnocratiser la question linguistique. [...] Il est temps de redonner la langue à la société civile, aux locuteurs 
ordinaires» (p.491-492). Il a cette belle phrase : «En fait, personne n'impose la norme, c'est la norme, résultat d'innombrables actes de langage et de jugements sur ces productions, qui s'impose.» (p.483) L'enjeu principal, que l'auteur partage avec les endogénistes, est la réduction de l'insécurité linguistique, mais elle ne peut passer, d'après lui, que par la reconnaissance, aux côtés du franbécois, du français standard international comme faisant partie de la langue en usage au Québec, réduisant les obstacles à l'intercompréhension entre francophones, et non par la normalisation des particularités du parlé québécois qui conduira les Québécois à l'isolement. Il milite pour la mise en œuvre de programmes de recherche visant à améliorer la connaissance du marché linguistique québécois, pour la refrancisation des formes anglicisées du français québécois (les anglicismes par calque notamment), pour renforcer la formation linguistique des enseignants.

La richesse de l'analyse sociologique et linguistique, la clarté du propos, le ton vif et énergique font de cet ouvrage un outil de réflexion très enrichissant, au-delà même de la seule société québécoise, et dont on peut recommander la lecture à tous les sociolinguistes.

Albane Geslin

Sciences Po Lyon, UMR Triangle

albane.geslin@sciencespo-lyon.fr 